\title{
Mechanisms of Edge-Localized-Mode Mitigation by External-Magnetic-Field Perturbations
}

\author{
M.Z. Tokar, ${ }^{1}$ T. E. Evans, ${ }^{2}$ A. Gupta, ${ }^{1}$ R. Singh,${ }^{3}$ P. Kaw,${ }^{3}$ and R. C. Wolf ${ }^{1}$ \\ ${ }^{1}$ Institut für Plasmaphysik, Forschungszentrum Jülich GmbH, Association FZJ-Euratom, 52425, Jülich, Germany \\ ${ }^{2}$ General Atomics, San Diego, California 92186-5606, USA \\ ${ }^{3}$ Institute for Plasma Research, Bhat, Gandhinagar-382428, India
}

(Received 7 September 2006; published 27 February 2007)

\begin{abstract}
Particle and energy transport in the tokamak edge transport barrier is analyzed in the presence of magnetic field perturbations from external resonant coils. In recent experiments such coils have been verified as an effective tool for mitigation of the edge-localized modes of type I. The observed reduction of the density in plasmas of low collisionality is explained by the generation of charged particle flows along perturbed field lines. The increase of the electron and ion temperatures in the barrier is interpreted by the reduction of perpendicular neoclassical transport with decreasing density and nonlocality of parallel heat transport. The found modification of the pressure gradient implies the stabilization of ballooning-peeling MHD modes responsible for type I ELMs.
\end{abstract}

DOI: 10.1103/PhysRevLett.98.095001

PACS numbers: 52.35.Py, 52.55.Fa

Introduction. -Edge-localized modes (ELMs) are an intrinsic feature of the high confinement $H$ mode in tokamaks [1]. On the one hand, they are beneficial by expelling from the plasma impurities produced at the machine walls. On the other hand, under reactor conditions usual type I ELMs would dramatically increase the erosion of divertor target plates and cause cyclic heat loads on them. Therefore, it is crucial to find a way to mitigate ELMs without negative consequences for the plasma. Recent experiments on the tokamak DIII-D [2] have demonstrated that large type I ELMs can be effectively mitigated without a significant loss of the confinement quality by applying resonant magnetic field perturbations from the so called $I$ coils. This mode of operation is highly desirable for future fusion reactors and it is very important to understand the physical mechanisms underlying the modification of plasma parameters through external field perturbations which leads to ELM suppression.

Up to now the increase of the heat transport in the barrier due to the magnetic field stochastization caused by the external coils has been considered [3] as the main cause of the ELM mitigation. By reducing the temperature at the barrier top this should lead to a decrease of the pressure gradient below the threshold for ballooning-peeling MHD modes considered normally as the cause of type I ELMs [4]. This approach, however, does not allow one to explain recent observations in DIII-D in plasma of very low collisionality [5], where a significant reduction of the plasma density but increase of the electron and ion temperatures in the barrier have been observed by activating $I$ coils. In the present Letter we demonstrate that these phenomena can be plausibly explained by taking into account (i) the modification of particle transport due to flows along perturbed magnetic field lines, (ii) the reduction of neoclassical perpendicular transport in the barrier with decreasing density, and (iii) the nonlocality of parallel heat transport intrinsic for plasma of low collisionality. Namely, the synergy of all these effects allows one to understand why the ELM can be mitigated by $I$ coils without a noticeable deterioration of the energy confinement in collisionless $H$-mode plasma.

Model for particle transport. - The effect of magnetic field perturbations from the $I$ coils on the transport of charged particles in the edge transport barrier is described by stationary continuity and parallel momentum equations in a plane geometry applicable at the plasma edge [6]:

$$
\begin{gathered}
\nabla_{\perp} \Gamma_{\perp}+\nabla_{\|} \Gamma_{\|}=k_{i} n n_{0}=-\frac{\partial J_{0}}{\partial r}, \\
m_{i}\left[\nabla_{\perp}\left(\frac{\Gamma_{\perp} \Gamma_{\|}}{n}\right)+\nabla_{\|}\left(\frac{\Gamma_{\|}^{2}}{n}\right)\right]=-\nabla_{\|}\left[n\left(T_{e}+T_{i}\right)\right],
\end{gathered}
$$

where $\Gamma_{\perp}$ and $\Gamma_{\|}$are the components of the plasma particle flux density in the directions perpendicular and parallel magnetic field lines; $r$ is the minor radius; $k_{i}$ is the ionization rate coefficient; $n$ and $n_{0}$ are the densities of charged particles and of neutrals recycling through the separatrix into the confinement volume; $J_{0}$ is the neutral flux density; $m_{i}$ is the ion mass; $T_{e}$ and $T_{i}$ are the electron and ion temperatures, respectively; plasma viscosity is neglected in Eq. (2).

By averaging over the surfaces of a constant $r$, which coincide with the magnetic surfaces without $I$-coil perturbations, one gets $\nabla_{\perp} \rightarrow \partial / \partial r$ and $\nabla_{\|} \rightarrow \alpha_{r} \partial / \partial r$, with $\alpha_{r} \ll 1$ being the averaged angle between perturbed field lines and magnetic surfaces. For a perturbation with a single Fourier harmonic $\alpha_{r}=0$. The $I$ coils produce, however, a broad spectrum of such harmonics and each of them generates a chain of magnetic islands instead of the resonant magnetic surface. When different island chains are overlapped, field line deviate stochastically in the radial 
direction and $\alpha_{r} \approx \sqrt{D_{\mathrm{FL}} / L_{K}}$, where $D_{\mathrm{FL}}$ and $L_{K}$ are the so-called field line diffusivity and Kolmogorov length [7]. Henceforth, we assume that $\alpha_{r}$ is constant in the whole edge barrier for the electron temperature with the width $\Delta_{b}$ and reduces to zero in the deeper plasma, $r<a-\Delta_{b}$, where $a$ is the minor radius of the separatrix; $\Delta_{b}$ is by a factor of 1.5 smaller than the width of the ion temperature barrier; see Fig. 4 in Ref. [5]. Under these assumptions Eqs. (1) and (2) can be integrated providing the following relationships for the radial components of the surface averaged perpendicular and parallel flux contributions, $\Gamma_{\perp}^{r} \approx \Gamma_{\perp}$ and $\Gamma_{\|}^{r} \approx \alpha_{r} \Gamma_{\|}$, respectively:

$$
\begin{gathered}
\Gamma_{\perp}^{r}+\Gamma_{\|}^{r}=J_{0}, \\
\left(\Gamma_{\|}^{r}\right)^{2}+\Gamma_{\perp}^{r} \Gamma_{\|}^{r}=\alpha_{r}^{2} n \frac{P_{b}-P}{m_{i}},
\end{gathered}
$$

with $P=n\left(T_{e}+T_{i}\right)$ being the plasma pressure and $P_{b}$ its value at the barrier top. From these equations $\Gamma_{\perp}^{r}$ and $\Gamma_{\|}^{r}$ can be determined and by assuming that the perpendicular flux is due to diffusion, $\Gamma_{\perp}=-D_{\perp} \partial n / \partial r$, we obtain the following equation describing the radial profile of the plasma density:

$$
\frac{d n}{d r}=-\frac{J_{0}}{D_{\perp}}+\frac{n}{J_{0}} \frac{\alpha_{r}^{2}}{D_{\perp}} \frac{P_{b}-P}{m_{i}},
$$

where the last contribution describes the effect of particle flows along perturbed field lines. Since the plasma pressure drops towards the separatrix, i.e., $P \leq P_{b}$, this contribution leads to a reduction of the density gradient for the same neutral flux and particle perpendicular diffusivity.

The transport of neutrals is described by taking into account that the rate coefficient for charge exchange with ions, $k_{\mathrm{cx}}$, exceeds significantly $k_{i}$. This results in a diffusive penetration of recycling neutrals into the plasma. The corresponding continuity equation for the neutral density $n_{0}$, see, e.g., Ref. [8], can be solved by the WKB method, providing in the first approximation for the neutral flux density

$$
J_{0}(r) \approx \Phi_{0} \exp \left[-\int_{r}^{a} \sqrt{k_{i}\left(k_{i}+k_{\mathrm{cx}}\right) \frac{m_{i}}{T_{i}}} n d r\right] .
$$

Without ELMs the influx of neutrals through the separatrix, $\Phi_{0}$, is mainly determined by the gas desorption from the machine walls and, therefore, does not change significantly by activating the $I$ coil.

Equation (5) has to be supplemented by the boundary condition for the density at the separatrix $n_{s}$. The latter is determined by the particle transport in the scrape-off layer (SOL) being a complex matter and out of the scope of the present Letter. Here we relate $n_{s}$ to its time averaged value without the $I$ coils, $n_{s}^{0}$, by taking into account that in the presence of the radial magnetic field from the coils the SOL radial width is increased with respect to its nominal value $\delta_{0}$ by a value of $\alpha_{r} L_{c}$, with $L_{c}$ being the SOL connection length. As a result the outflow of plasma particles through the separatrix, which in a steady state is equal to $\Phi_{0}$, has to be transported to the divertor plate in a thicker region. Therefore the density at the separatrix with the $I$ coils one can roughly estimate as follows:

$$
n_{s} \approx \frac{n_{s}^{0} \delta_{0}}{\delta_{0}+\alpha_{r} L_{c}} .
$$

Finally, the density at the barrier top, $n_{b}$, involved into $P_{b}$ is determined by requiring that $n\left(a-\Delta_{b}\right)$ obtained from the integration of Eq. (5) has to coincide with $n_{b}$. By a numerical solution of Eq. (5) this is achieved in an iterative procedure.

An analytical estimate for $n_{b}$ can be obtained by approximating $d n / d r$ at the separatrix, $r=a$, as $\left(n_{s}-\right.$ $\left.n_{b}\right) / \Delta_{b}$. Then Eq. (5) leads to

$$
n_{b}=\frac{\Phi_{0}+\left(\frac{D_{\perp}}{\Delta_{b}}+\frac{T_{e s}+T_{i s}}{\Phi_{0} m_{i}} \frac{n_{s}^{0} \delta_{0} \alpha_{r}^{2}}{\delta_{0}+\alpha_{r} L_{c}}\right) \frac{n_{s}^{0} \delta_{0}}{\delta_{0}+\alpha_{r} L_{c}}}{\frac{D_{\perp}}{\Delta_{b}}+\frac{T_{e b}+T_{i b}}{\Phi_{0} m_{i}} \frac{n_{s}^{0} \delta_{0} \alpha_{r}^{2}}{\delta_{0}+\alpha_{r} L_{c}}}
$$

with $T_{e b}, T_{i b}$ being the temperatures at the pedestal top and $T_{e s}, T_{i s}$-at the separatrix. One can see that the denominator in Eq. (7) is monotonously increasing function of $\alpha_{r}$. On the contrary, the numerator decreases with growing $\alpha_{r}$ when this is either small or large enough. In general, the barrier density decreases with $\alpha_{r}$. This tendency is enhanced by the increase of the temperatures in the barrier with the $I$ coils, an unexpected phenomenon observed in the $H$-mode transport barrier of low collisionality. A possible physical mechanism, being responsible for this, is discussed in the next section.

Model for heat transport. - The heat flux density in the radial direction $q_{r}$ includes contributions from the heat conductivity perpendicular to the magnetic field $\kappa_{\perp}$ and along the perturbed field lines $\kappa_{\|}$:

$$
q_{r} \approx-\kappa_{\perp} \nabla_{\perp} T-\kappa_{\|} \alpha_{r} \nabla_{\|} T .
$$

In the edge transport barrier where plasma instabilities, driving anomalous transport, are suppressed, $\kappa_{\perp}$ can be described by the neoclassical theory [9]. This has been validated by interpretive [10] and predictive [11] transport modeling of $H$-mode plasmas. In plasma of low collisionality the so-called "banana" regime of neoclassical transport is of relevance and $\kappa_{\perp} \sim n^{2}$. Thus we have to expect a reduction of this contribution to the total heat conductivity with external magnetic perturbations resulting from a decrease of the plasma density in the barrier. One may, however, think that this effect can be easily washed out through the activation of the parallel heat conduction channel. To understand why this does not happen, at least not to a significant extent, we analyze the accompanying behavior of $\kappa_{\|}$. 
Normally the Spitzer-Härm formula is used for $\kappa_{\|}$: $\kappa_{\|}^{\mathrm{SH}}=\xi_{\mathrm{SH}} n T \tau / m$, with $\xi_{\mathrm{SH}} \approx 3$ and $\tau$ being the time between Coulomb collisions. Since $\kappa_{\|}^{\mathrm{SH}} \sim T^{5 / 2}$, an extremely high parallel transport has to be expected in hot collisionless plasma. However, $\kappa_{\|}^{\mathrm{SH}}$ has been deduced in the limit of a very small mean free path length between collisions, $\lambda \approx \tau \sqrt{T / m}$, compared to the characteristic dimension for the temperature change along field lines, $L_{T}=1 /\left|\nabla_{\|} \ln T\right|$. In the opposite collisionless limit, $\lambda \gg$ $L_{T}$, a free-streaming heat flux, $q_{\mathrm{FS}}=n T^{3 / 2} / \sqrt{m}$, may be expected $[12,13]$. However, an interpretation of laser fusion experiments led to the conclusion that this heat flux limit has to be strongly reduced by the factor $0.03 \leq \xi_{\mathrm{FS}} \leq$ 0.1 [14]. Later numerical Fokker-Planck simulations of laser produced plasmas [15] provided $0.072 \leq \xi_{\mathrm{FS}} \leq 0.1$ and similar values have been obtained by modeling of tokamak scrape-off layer plasmas with strong recycling [16]. Two physical mechanisms responsible for such a strong reduction of the heat flux limit with respect to $q_{\mathrm{FS}}$ have been elucidated. First, nonlocal effects in collisionless plasma reduce the perturbation in the distribution function caused by the temperature gradient [17]. Namely, this perturbation results in heat conduction. Second, light electrons are braked by the ambipolar electric field [12]. Therefore $\xi_{\mathrm{FS}}$ is by a factor of 3 smaller for them than for heavy ions which are accelerated. A smooth transition between collisional and collisionless limits can be described by the formula [14]

$$
\kappa_{\|} \approx \kappa_{\|}^{\mathrm{SH}} /\left(1+\frac{\xi_{\mathrm{SH}}}{\xi_{\mathrm{FS}}} \frac{\lambda}{L_{T}}\right),
$$

and Eq. (8) results in the following:

$$
q_{r} \approx-\left(\kappa_{\perp}+\frac{\kappa_{\|}^{\mathrm{SH}} \alpha_{r}^{2}}{1+\alpha_{r} \frac{\xi_{\mathrm{SH}}}{\xi_{\mathrm{FS}}} \frac{\lambda}{T}\left|\frac{\partial T}{\partial r}\right|}\right) \frac{\partial T}{\partial r} .
$$

By replacing $\partial T / \partial r$ with $-T_{b} / \Delta_{b}$, one finds that for the conditions in question, $n_{b} \approx(1-2) \times 10^{19} \mathrm{~m}^{-3}, T_{b} \approx$ $1 \mathrm{keV}, \alpha_{r} \geq 10^{-4}$, the second contribution in the denominator of Eq. (10) is much larger than the first one. By taking into account that the power lunched into the discharge does not change by the I-coil activation, we obtain for the banana regime of neoclassical transport:

$$
\frac{\partial \ln T_{b}}{\partial \ln \alpha_{r}} \sim\left(1+\alpha_{\perp}\right) \frac{\partial \ln \left(1 / n_{b}\right)}{\partial \ln \alpha_{r}}-1+\alpha_{\perp}
$$

with $\alpha_{\perp}=\frac{\kappa_{\perp} T_{b}}{q_{r} \Delta_{b}}$ being the fraction of perpendicular heat conduction in the total radial heat flux. For sufficiently large perturbations Eq. (7) predicts $n_{b} \sim 1 / \alpha_{r}$ and $T_{b}$ has to increase with $\alpha_{r}$, i.e., with the current in the $I$ coils, in agreement with observations.

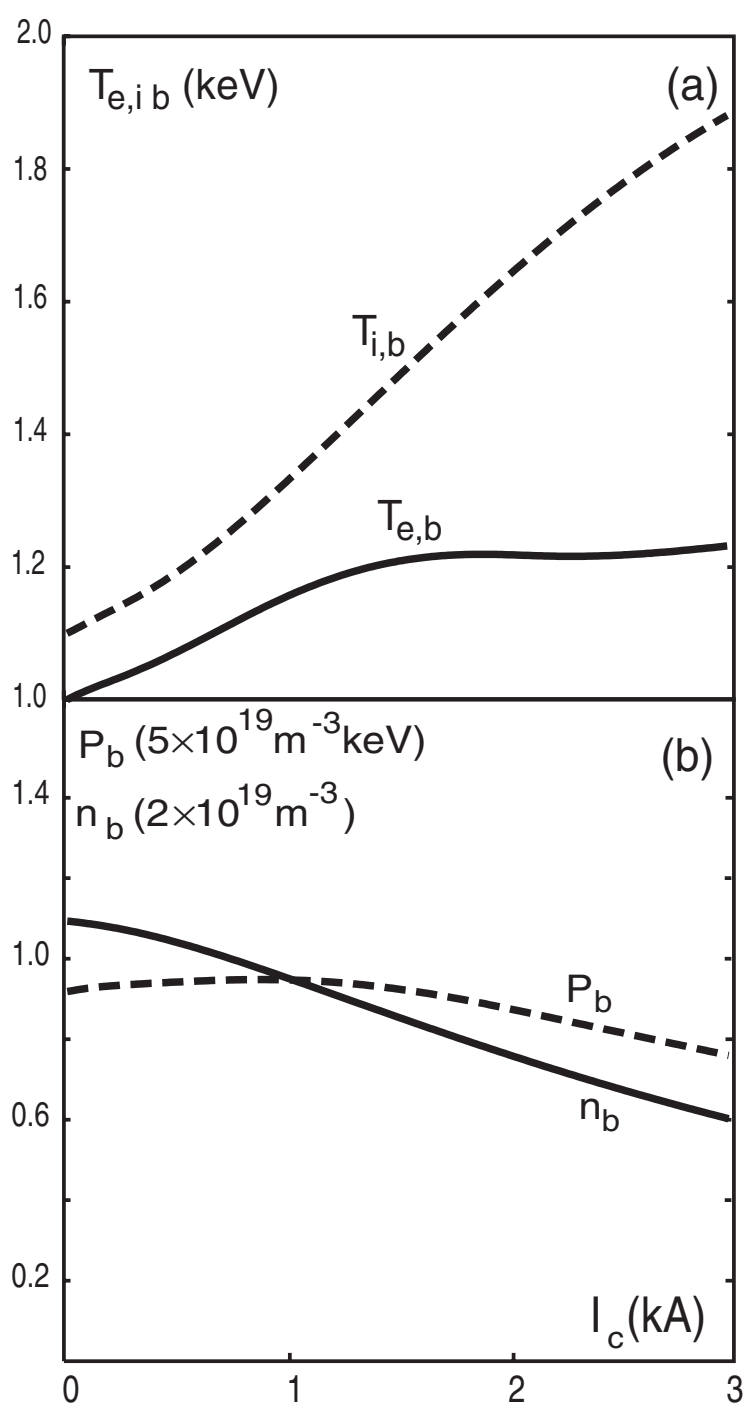

FIG. 1. Variation of the ion and electron temperatures (a), plasma density and pressure (b) at the top of the electron temperature pedestal with the current in $I$ coils.

Numerical modeling of parameter profiles. - The qualitative consideration above has been validated by numerical solution of transport equations. The reference values of perpendicular heat conduction in the barrier at the discharge stage without the $I$ coils have been estimated from the time averaged plasma parameter profiles and total heating power, the charged particle diffusivity, and neutral influx - from UEDGE modeling [5]. Figure 1(a) displays the calculated dependence on the $I$-coil current $I_{c}$ of the electron and ion temperatures, $T_{e b}$ and $T_{i b}$, at the top of the electron temperature barrier of the width $\Delta_{b} \approx 0.7 \mathrm{~cm}$. The radial magnetic field $b_{r}$ needed to calculate $D_{\mathrm{FL}}$ and $\left\langle\alpha_{r}\right\rangle$ varies linearly with $I_{c}$. For the perturbation Fourier harmonics with the poloidal and toroidal mode numbers $m / n=(11-13) / 3$, a current of $3 \mathrm{kA}$ corresponds to $b_{r} / B=2.6 \times 10^{-4}$ with $B$ being the total magnetic field 


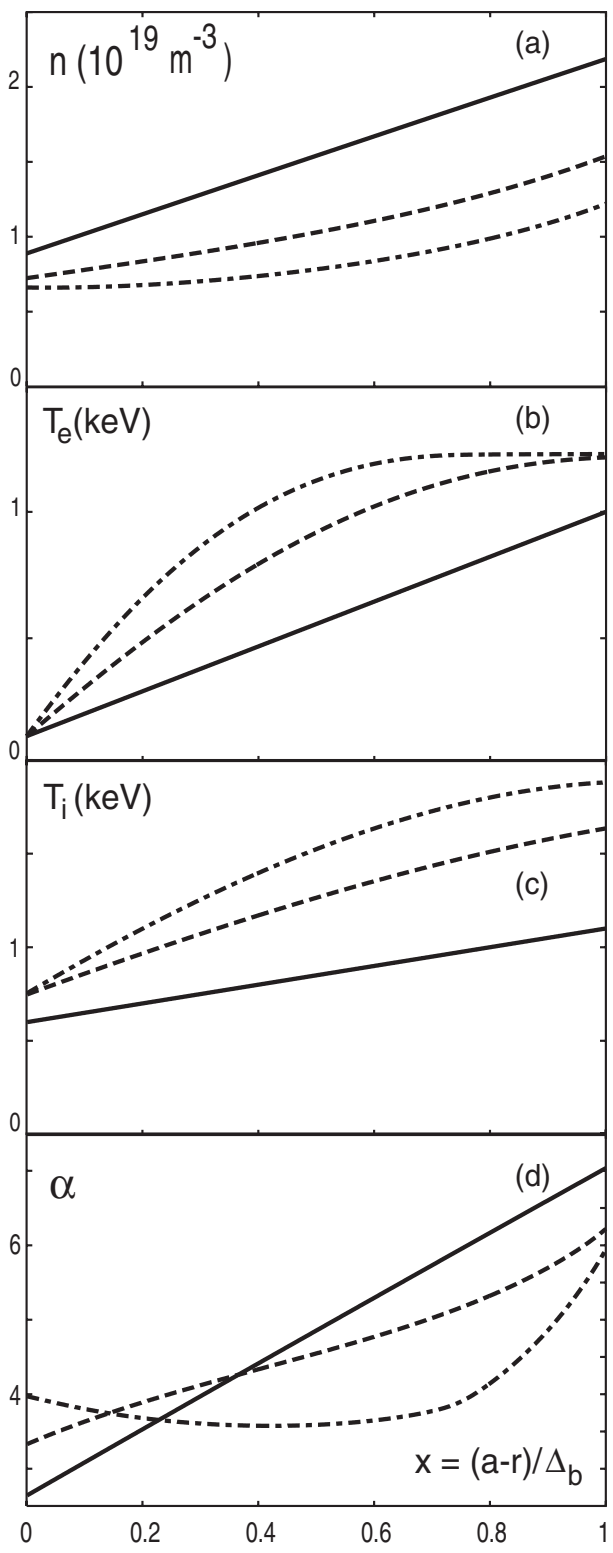

FIG. 2. Computed radial profiles of the plasma density (a), electron temperature (b), ion temperature (c), and normalized pressure gradient (d) without I coils (solid curves), with the I-coil current of $2 \mathrm{kA}$ (dashed curves), and $3 \mathrm{kA}$ (dash-dotted curves).

of $2 T$. For the same temperatures the parallel heat transport of ions is a factor of $\sqrt{m_{e} / m_{i}}$ smaller than that of electrons. Therefore, it by far cannot compensate the drop of $\kappa_{\perp}^{i}, 1-$ $\alpha_{\perp}^{i} \ll 1-\alpha_{\perp}^{e}$ in Eq. (11) and the increase of $T_{i b}$ with the $I$-coil current is significantly larger than that of $T_{e b}$ since Coulomb collisions are too rare and ineffective for tem- perature coupling. In the total plasma pressure $P_{b}=$ $n_{b}\left(T_{e b}+T_{i b}\right)$, the temperature growth compensates to a large extent the drop of the plasma density; see Fig. 1(b). The radial profiles of the plasma density, electron and ion temperatures, and the normalized pressure gradient, $\alpha=$ $\frac{2 \mu_{0} q^{2} R}{B^{2}}\left|\frac{d P}{d r}\right|$, with $q$ and $R$ being the safety factor and the major plasma radius, within the electron transport barrier are presented in Fig. 2 for $I_{c}=0,2 \mathrm{kA}$, and $3 \mathrm{kA}$. One can see the significant decrease of the maximum $\alpha$ level with the $I$ coils. The latter happens due to approaching of the pressure profile to a linear one with a constant $\alpha$. This is caused by the behavior of both density and temperature profiles. (The sharp increase of $\alpha$ in the region $0.8 \leq x \leq$ 1 is due to the assumption that stochastization is absent beyond the electron transport barrier, $x \geq 1$, which is probably unrealistic and has to be removed in a future more sophisticated study). The reduction of $\alpha$ results also in the decrease of the dominant bootstrap contribution to the plasma current in the edge transport barrier. As a result, the plasma operation point moves into the region of peeling-ballooning stability and ELMs are mitigated.

This study has been partly performed within GRK1203 of German Research Society.

[1] F. Wagner et al., Phys. Rev. Lett. 49, 1408 (1982).

[2] T. E. Evans et al., Phys. Rev. Lett. 92, 235003 (2004).

[3] M. Bécoulet et al., 20th IAEA Fusion Energy Conference, Vilamoura, Portugal, 2004 (International Atomic Energy Agency, Vienna, 2004), TH/1-3rc.

[4] P. B. Snyder et al., Phys. Plasmas 9, 2037 (2002).

[5] T. E. Evans et al., Nature Phys. 2, 419 (2006).

[6] P. C. Stangeby, The Plasma Boundary of Magnetic Fusion Devices (Institute of Physics Publishing, Bristol, 2000).

[7] Ph. Ghendrih, A. Grosman, and H. Capes, Plasma Phys. Controlled Fusion 38, 1653 (1996).

[8] M.Z. Tokar, Phys. Rev. Lett. 91, 095001 (2003).

[9] J. Wesson, Tokamaks (Claredon Press, Oxford, 2004), 3rd ed.

[10] A. Rogister et al., Nucl. Fusion 42, 1144 (2002).

[11] D. Kalupin et al., Nucl. Fusion 45, 468 (2005).

[12] R. W. Harvey et al., Phys. Rev. Lett. 47, 102 (1981).

[13] A. B. Rechester and M. N. Rosenbluth, Phys. Rev. Lett. 40, 38 (1978).

[14] R. C. Malone, R. L. McCrory, and R. L. Morse, Phys. Rev. Lett. 34, 721 (1975).

[15] J. F. Luciani, P. Mora, and J. Virmont, Phys. Rev. Lett. 51, 1664 (1983).

[16] R. Chodura, Contrib. Plasma Phys. 32, 219 (1992).

[17] E. M. Epperlein and R. W. Short, Phys. Fluids B 4, 2211 (1992). 